\title{
Usos de porque en el habla infantil: Implicaciones del desarrollo de la complejidad sintáctica ${ }^{1}$
}

\author{
Iván Enríquez Martínez - Universidade de Santiago de Compostela \\ ivan.enriquez.martinez@usc.es
}

Rebut / Received: 26-10-17

Acceptat / Accepted: 19-2-18

Resum. Usos de porque en la parla infantil: Implicacions del desenvolupament de la complexitat sintàctica. El procés d'adquisició i desenvolupament de les construccions complexes en la parla infantil és un tema d'interès creixent en la bibliografia especialitzada, tant per les seves implicacions teòriques com pràctiques. En aquest article es presenta un estudi longitudinal que pretén descriure l'emergència i les funcions del connector porque entre els 2; 0 i els 4; 0 anys, la qual cosa permet conèixer l'origen ontogènic de les construccions complexes de tipus causal. S'han examinat produccions espontànies emeses per un total de deu participants procedents del corpus Koiné de parla infantil. Els resultats, coincidents amb els obtinguts en altres contextos idiomàtics, indiquen que porque és un connector primerenc que es caracteritza pels seus valors pragmaticodiscursius i que apareix fonamentalment en estructures coconstruïdes amb l'interlocutor adult. Aquestes característiques d'ús han de ser tingudes en compte a l'hora d'elaborar materials pedagògics, així com per conformar perfils de desenvolupament típic.

Paraules clau: parla infantil, desenvolupament gramatical, complexitat sintàctica, construccions causals, connector porque.

Abstract. Uses of porque in child language: implications of the development of syntactic complexity. The acquisition and development of complex sentences in child language is a topic of growing interest within specialized literature for its theoretical and practical implications. In this paper, we introduce a longitudinal study focused on the emergence and functions that the

1. Este trabajo se inscribe en el marco del proyecto Adquisición fónica y corpus. Tratamiento en Phon del corpus Koiné de habla infantil (FFI2017-82752-P), financiado por el Ministerio de Economía, Industria y Competitividad y desarrollado por el grupo Koiné (www.usc.es/koine). 
Spanish connector porque presents in the talk of children aged between 2;0 and 4;0 years. This will help to understand the ontogenetic origin of causal constructions. We have analyzed the spontaneous productions of ten participants from the Koiné corpus. The results are similar to those obtained in other languages and they prove that porque is an early connector characterized by its pragmatic-discursive function and its appearance in cross-turn shared constructions. These particular features of use could be taken into account for both elaborating pedagogical materials and defining typical development profiles.

Keywords: child language, grammatical development, syntactic complexity, causal constructions, Spanish connector porque.

\section{Introducción}

El proceso de adquisición y desarrollo de la lengua materna ha sido uno de los temas centrales de la lingüística en las últimas décadas. Sin embargo, la cuestión de la complejidad sintáctica no ha sido tratada con la debida profundidad. Aunque su definición difiere en función de la perspectiva teórica adoptada por cada autor, lo cierto es que la bibliografía especializada suele otorgar un papel clave a las relaciones interclausulares y a los conectores que las expresan (Alfaro, Crespo y Alvarado, 2016). El propósito del presente trabajo es examinar los primeros usos de uno de los nexos más frecuentes del español, porque, partiendo de los datos proporcionados por un corpus de habla infantil espontánea y poniendo especial énfasis en las propiedades de las construcciones en que aparece. En última instancia, se pretende aportar una descripción de sus funciones en niños con desarrollo típico, de modo que resulte útil a la hora de confeccionar materiales clínicos y pedagógicos en lengua española (Fernández Pérez, 2014).

\section{Usos tempranos de porque: estado de la cuestión}

Si bien la adquisición del lenguaje ha sido uno de los temas principales de la lingüística desde mediados del siglo XX, puede afirmarse que todavía quedan parcelas por transitar. En concreto, se ha destacado que el desarrollo de las construcciones complejas se ha estudiado, sobre todo, desde una perspectiva formal y se ha descuidado su uso espontáneo en las edades tempranas (Tomasello, 2003). Además, sabemos que la aparición de los primeros conectores es un fenómeno ligado al desarrollo de determinadas habilidades pragmáticas que resultarán fundamentales en los primeros años de escolarización de los niños, como la capacidad narrativa (Sebastián y Slobin, 1994).

A raíz de esta necesidad del campo, los nuevos enfoques basados en el uso, de inspiración constructivista, se han interesado por la cuestión y han cosechado interesantes resultados en esta parcela del desarrollo gramatical. Entre otros aspectos, la teoría basada 
en el uso defiende que la adquisición de las construcciones complejas no difiere, en esencia, del proceso de desarrollo de los recursos morfosintácticos básicos y que se produce mediante un proceso gradual en el que el contexto discursivo y el interlocutor desempeñan un papel relevante (Diessel, 2004; Givón, 2009; Tomasello, 2003). Por ello, los conectores tempranos manifiestan en un principio funciones pragmáticas, actuando como marcadores discursivos, y solamente en un momento posterior serán utilizados como enlaces intraoracionales (Aguado, 1995). En español, esta idea ha sido corroborada mediante datos de corpus y con diferentes partículas - como y (Prego Vázquez, 2006) o pero (Varela, 2011)—, cuya aparición se sitúa entre los 2;0 y los 3;0 ańos. A estas edades, pues, comienzan a desarrollarse las primeras construcciones complejas en español (Aparici, Capdevila, Serrat y Serra, 2001).

En cuanto a los conectores causales, los datos disponibles para lenguas como el inglés (Braunwald, 1997; Diessel, 2004), el francés (Sekali, 2012), el neerlandés (EversVermeul y Sanders, 2011) o el alemán (van Veen, Evers-Vermeul, Sanders y van den Bergh, 2009) apuntan a un dominio relativamente temprano, pues comienzan a ser utilizados antes de los 3;0 años, siempre con posterioridad a otros enlaces considerados cognitivamente más sencillos, como los aditivos (Bloom, Lahey, Hood, Lifter y Fliess, 1980). En español la situación es similar, puesto que la bibliografía sitúa la aparición de porque poco después de los 2;0 años (Hernández-Pina, 1984). Ahora bien, además de la emergencia de la forma, hemos de tener presente sus diferentes funciones, de manera que Aguado (1988), que encuentra su uso plenamente asentado a los 2;6 ańos, destaca que en el habla infantil este conector se caracteriza por sus valores expletivos:

Respecto a las oraciones introducidas por la conjunción porque, las menos están contabilizadas como oraciones subordinadas, ya que dicha conjunción, en muchos casos, sirve como comodín y/o como una forma de responder, que se antepone a una oración principal (Aguado, 1988, p. 82).

Este carácter predominantemente pragmático, corroborado en modernos estudios de corpus, tanto en español (Díez-Itza y Pérez Toral, 2001) como en otras lenguas (Diessel, 2004; Sekali, 2012), tiene implicaciones en su desarrollo como enlace interclausular. Así, Prego Vázquez (2010), profundizando en sus valores pragmático-discursivos, identifica una tendencia evolutiva en la que se van incorporando gradualmente, y por este orden, sus funciones interaccional, pragmática e ideativa, si bien destaca que en las edades tempranas porque actúa sobre todo como marcador en el plano del intercambio comunicativo (Prego Vázquez, 2010, p. 198), unos resultados comparables a los obtenidos por Evers-Vermeul y Sanders (2011) en neerlandés. Por ello, hemos de poner en relación esta aportación con el estudio de Aparici et al. (2001), quienes encontraron que las oraciones causales con porque tienen un origen interaccional, puesto que los niños se apoyan inicialmente en los enunciados de sus interlocutores antes de producir ambas cláusulas por sí mismos. 
La bibliografía señala, por tanto, que las construcciones con porque se desarrollan mediante un patrón evolutivo de integración, en el que dos enunciados independientes acaban dando paso a una única construcción constituida por dos conjuntos verbales. Lo interesante es que esta tendencia, en la que las estructuras causales se desarrollan a través de un proceso recíproco en el discurso, ha sido detectada por Braunwald (1997) o Diessel (2004) en inglés, así como por Sekali (2012) en francés ${ }^{2}$. Es más, la adquisición de las adversativas en español parece seguir esta misma ruta de acuerdo con Varela (2011), autora que, distinguiendo diferentes construcciones en función del grado de implicación del interlocutor adulto, constató que pero se incorpora como nexo gramatical en el diálogo adulto-niño y a partir de un nicho discursivo concreto. De hecho, investigaciones recientes centradas en el inglés, como el trabajo de Givón (2009), subrayan la importancia evolutiva que las llamadas construcciones compartidas tienen en las fases tempranas. Diessel (2004, pp. 171-173) va un paso más allá y habla, incluso, de la influencia del input parental en la adquisición de los conectores, idea demostrada en estudios como los de van Veen et al. (2009, 2013), si bien destacan que su incidencia es diferente a lo largo del desarrollo.

Así pues, partiendo de estas dos grandes ideas —incorporación del conector en el contexto dialógico y prevalencia de los valores pragmáticos-, nuestra intención es caracterizar las primeras estructuras con porque para seguir profundizando en su proceso de desarrollo como nexo interclausular. Para ello, como se explica en el siguiente apartado, examinaremos qué forma presentan las construcciones infantiles en que aparece, siguiendo una metodología similar a la empleada por Varela (2011), y qué tipo de causalidad expresan, siempre teniendo en cuenta los precedentes pragmáticodiscursivos del enlace. Consideramos que este enfoque permite realizar una adecuada descripción del origen y desarrollo temprano de las construcciones complejas causales.

\section{Metodología}

El propósito del presente trabajo es realizar un estudio longitudinal, de carácter descriptivo, que establezca las funciones principales de porque entre los 2;0 y los 4;0 años y, al tiempo, se caractericen el tipo de estructuras en que aparece. Para cumplir con este objetivo decidimos recurrir a muestras orales, extraídas de un corpus lingüístico. En concreto, se ha manejado el corpus Koiné, que se integra en la conocida base de datos internacional CHILDES (Díez-Itza, Snow y MacWhinney, 1999). Este repertorio proporciona producciones espontáneas recogidas en escuelas infantiles de Galicia mediante técnicas de observación participante. Aunque el corpus abarca un periodo de seguimiento total que va desde los 1;6 hasta los 4;5 años, las franjas intermedias (esto es,

2. No obstante, no todos los autores se muestran de acuerdo con esta idea. Por ejemplo, Bloom et al. (1980) defienden que los niños no utilizan los conectores para vincular discursivamente sus enunciados con los de su interlocutor, sino sobre todo para cohesionar sus propios enunciados. 
2;6-3;0 y 3;0-3;6) son las que mayor representación tienen (Fernández Pérez, 2011, pp. 11-36), por lo que resulta particularmente apropiado para nuestros intereses.

\section{1. Participantes}

El tamańo del corpus Koiné, constituido por 166 transcripciones que recogen más de 50 horas de interacción y en las que intervienen hasta 66 participantes, exigía realizar una selección que asegurara una cierta profundidad en el examen de los datos. Los participantes examinados en este trabajo fueron elegidos en virtud de diferentes criterios como su edad, su sexo o su nivel de desarrollo gramatical ${ }^{3}$. En este mismo sentido, era necesario que los nińos hubiesen recibido un seguimiento prolongado, superior a los doce meses, en el periodo que conduce de los 2;0 a los 4;0 ańos.

Por otro lado, era necesario que los niños seleccionados presentaran una participación activa y regular en el corpus, de modo que se calculó un índice basado en el número de producciones emitidas y el tiempo total de las sesiones de grabación en que intervienen. A pesar de que existen diferencias tanto en la franja cronológica cubierta como en el número de enunciados, consideramos que ello no condiciona los resultados obtenidos en el estudio. De hecho, la aplicación del test Shapiro-Wilk $(p=0.05)$, teniendo en cuenta el índice de participación, confirma que la distribución de los datos es normal (W=0.927; p-value=0.42).

En definitiva, los criterios mencionados nos han permitido trabajar con una muestra de 10 (diez) participantes balanceada en lo que respecta al sexo y que presentamos en la Tabla 1.

Estos diez niños, como todos los que participaron en el corpus Koiné, presentaban un desarrollo cognitivo y lingüístico dentro de los márgenes habituales, tal y como confirman las cifras de la Longitud Media del Enunciado (LME) ${ }^{4}$ (Brown, 1973). Podemos afirmar, por tanto, que estamos ante una muestra de desarrollo típico que, en principio, pretende ser representativa del periodo que conduce de los 2;0 a los 4;0 años en un contexto bilingüe español-gallego 5 .

3. Dado que las grabaciones se realizaron en un contexto bilingüe, no todos los participantes podían ser objeto de análisis, sino solamente aquellos que utilizaran preferentemente el castellano durante las sesiones. No obstante, conviene tener en cuenta que los nińos han crecido en un contexto bilingüe, si bien creemos que ello no influye en el curso evolutivo de porque, ya que conceptual, funcional y categorialmente ambas partículas son prácticamente idénticas en castellano y en gallego. Sobre las particularidades del desarrollo lingüístico en situaciones de bilingüismo puede consultarse Hoff (2014, pp. 261-292).

4. La LME es la adaptación al español de la Mean Length of Utterances (MLU), la medida más extendida en el campo para calcular el nivel de desarrollo gramatical de los niños y, por ende, su edad lingüística (Brown, 1973). Aunque en inglés se calcula en morfemas, en español se recomienda hacerlo en términos léxicos (Clemente, 1989), tal y como hemos hecho.

5. Los creadores del corpus Koiné certificaron, antes de iniciar las grabaciones, que los participantes no presentaban ninguna patología que pudiera afectar a su desarrollo comunicativo; además, las sesiones se 
Tabla i. Muestra de ANÁLisis

\begin{tabular}{|l|c|c|c|c|c|c|}
\hline Participante & Sexo & $\begin{array}{c}\text { LME } \\
\text { inicial }\end{array}$ & Sesiones & Enunciados & $\begin{array}{c}\text { Índice de } \\
\text { participación }\end{array}$ & Periodo \\
\hline ANP & $\mathrm{f}$ & 1,83 & 19 & 1178 & 5,13 & $\begin{array}{c}2 ; 1.12- \\
3 ; 9.09\end{array}$ \\
\hline CEC & $\mathrm{f}$ & 1,40 & 12 & 674 & 3,82 & $\begin{array}{c}1 ; 10.14 \\
-3 ; 1.20\end{array}$ \\
\hline ELI & $\mathrm{f}$ & 1,89 & 12 & 458 & 2,68 & $\begin{array}{c}2 ; 6.14- \\
4 ; 3.21\end{array}$ \\
\hline IAG & $\mathrm{m}$ & 1,25 & 28 & 1483 & 2,70 & $\begin{array}{c}1 ; 11.24 \\
-4 ; 1.08\end{array}$ \\
\hline JOR & $\mathrm{m}$ & 1,67 & 22 & 1518 & 4,68 & $\begin{array}{c}1 ; 11.23 \\
-3 ; 7.08\end{array}$ \\
\hline NER & $\mathrm{f}$ & 1,17 & 12 & 562 & 2,57 & $\begin{array}{c}2 ; 3.10- \\
3 ; 6.23\end{array}$ \\
\hline PAU & $\mathrm{f}$ & 1,56 & 14 & 380 & 1,83 & $\begin{array}{c}2 ; 6.15- \\
3 ; 9.15\end{array}$ \\
\hline RIC & $\mathrm{m}$ & 1,32 & 23 & 1555 & 4,00 & $\begin{array}{c}1 ; 10.18- \\
3 ; 11.14\end{array}$ \\
\hline XAC & $\mathrm{m}$ & 1,86 & 22 & 929 & 2,72 & $\begin{array}{c}2 ; 5.10- \\
4 ; 0.12\end{array}$ \\
\hline XUN & $\mathrm{m}$ & 1,75 & 16 & 1438 & 2,75 & $\begin{array}{c}2 ; 0.08- \\
3 ; 5.29\end{array}$ \\
\hline
\end{tabular}

\section{2. Procedimiento}

Dado que los datos han sido tomados de un corpus de habla infantil, la principal tarea que nos ocupó en la elaboración de este estudio fue el de localizar, extraer y codificar todos los usos de porque encontrados. Para ello, se recurrió al paquete de programas informáticos CLAN (MacWhinney, 2000), que ha sido especialmente diseñado para el análisis de transcripciones con formato CHAT, que comparten todos los corpus alojados en el sistema CHILDES. Además de MLT, con el que se calculó la LME y el número de enunciados de cada niño, se manejaron fundamentalmente dos programas más del paquete CLAN: KWAL, que extrae las ocurrencias en contexto, en miras a realizar un

programaron en varias escuelas infantiles ubicadas en diferentes entornos, procurando así que la extracción social de los niños fuese diversa (v., para más información, Fernández Pérez, 2011, pp. 22-25).

6. Como hemos adelantado, para calcular este índice de participación de los niños en el corpus se ha dividido el número de enunciados totales entre el tiempo que suman todas las grabaciones en que participan $(=3,59 ; \mathrm{S}=1,29 ; \mathrm{SE}=0,45 ; \mathrm{IC}=2,43-4,73)$. 
análisis cualitativo, y FREQ, el más importante desde el punto de vista cuantitativo, puesto que permite contabilizar el número de usos de porque y calcular la distribución de sus funciones, dado que el corpus seleccionado contiene una línea de codificación morfosintáctica (Fernández Pérez, 2011, pp. 22-36). Estas han sido las técnicas de procesamiento de los datos que hemos manejado para conocer cuándo y cómo se utiliza porque en el habla infantil.

\section{3. Análisis}

Además de establecer, mediante una media aritmética, en qué momento se localizan los primeros usos de porque, nos interesaba profundizar en sus funciones y caracterizar las construcciones en que aparece. Por este motivo, aparte de emplear técnicas cuantitativas de carácter descriptivo, procedimos a realizar un análisis cualitativo de todas las ocurrencias detectadas. Así, en primer lugar se calculó la distribución de las dos grandes funciones, pragmática y sintáctica, en las muestras. A continuación, se examinaron aspectos propios de ambas dimensiones: tipo de cohesión y uso en pares de adyacencia pregunta-respuesta, por un lado, y propiedades de las construcciones y tipo de causalidad expresada, por el otro.

Toda esta información, que aúna aspectos pragmático-discursivos y gramaticales, permite, partiendo de la propuesta realizada por Varela (2011), distinguir varios tipos de construcciones infantiles, que resumimos en la Tabla 2.

TABla 2. Tipos De CONSTRUCCIONES INFANTILES CON PORQUE

\begin{tabular}{|c|c|c|c|}
\hline $\begin{array}{c}\text { Tipo de } \\
\text { construcción }\end{array}$ & $\begin{array}{c}\text { Estrategia de } \\
\text { producción }\end{array}$ & $\begin{array}{c}\text { Unidades } \\
\text { conectadas }\end{array}$ & $\begin{array}{c}\text { Función de } \\
\text { porque }\end{array}$ \\
\hline $\begin{array}{c}\text { Construcciones } \\
\text { compartidas }\end{array}$ & Compartida & Turnos & $\begin{array}{c}\text { Conector } \\
\text { pragmático }\end{array}$ \\
\hline $\begin{array}{c}\text { Construcciones } \\
\text { individuales }\end{array}$ & Individual & $\begin{array}{c}\text { Intervenciones } \\
\text { / Enunciados }\end{array}$ & $\begin{array}{c}\text { Conector } \\
\text { pragmático }\end{array}$ \\
\hline $\begin{array}{c}\text { Construcciones } \\
\text { integradas }\end{array}$ & Individual & $\begin{array}{c}\text { Unidades } \\
\text { gramaticales } \\
\text { (cláusulas) }\end{array}$ & Conector sintáctico \\
\hline
\end{tabular}

Así pues, hemos codificado todas las ocurrencias de porque partiendo de tres grandes categorías de análisis, que se diferencian entre sí por tres parámetros fundamentales: la estrategia de producción, el tipo de unidades vinculadas — partiendo de la clasificación de unidades conversacionales propuesta por Briz (2000) — y la función del conector.

7. Conviene aclarar que no se computaron aquellos usos localizados en contextos de omisión parcial y que las producciones balbuceantes fueron contabilizadas como una única ocurrencia. 
Consideramos que este sistema categorial resulta particularmente útil para el análisis del desarrollo de las construcciones complejas desde una perspectiva dialógica.

Así, y en primer lugar, las construcciones compartidas están constituidas por dos proposiciones codificadas en turnos diferentes, de tal modo que el segundo, emitido por el niño, está encabezado por porque y actúa como conjunto dependiente de la producción previa, emitida por otro interlocutor, generalmente un adulto, tal y como se puede comprobar en el ejemplo (1).

(1) Transcripción: elf4_01 (línea 84). Participante: IAG. Edad: 3;6.08.

*MON: se subió por aquí .

*IAG: porque no se lastima .

En este tipo de construcciones, por tanto, la estrategia de producción es compartida y porque, que actúa como conector pragmático, vincula turnos diferentes.

Por otro lado, las construcciones individuales, como la que tenemos en el ejemplo (2), son estructuras de producción individual, ya que las dos proposiciones entre las que se expresa una relación causal son codificadas por el niño. No obstante, porque sigue actuando a nivel discursivo, como conector pragmático, puesto que vincula unidades conversacionales diferentes, sean intervenciones o enunciados, cada una con su propio contorno entonativo.

(2) Transcripción: mil2_05 (línea 286). Participante: NER. Edad: 3;6.23

${ }^{*}$ NER: a ver si lo aprende .

${ }^{*} \mathrm{MON}$ : eso .

*NER: porque yo [/] yo ya aprendí .

Por último, las construcciones integradas responden al modelo prototípico de construcciones causales en las gramáticas de la lengua adulta, como se puede comprobar en el ejemplo (3). Así, aunque, como en el caso anterior, su estrategia de producción es individual, en este caso las dos proposiciones se codifican ya en la misma unidad melódica, de manera que porque vincula dos unidades gramaticales (dos cláusulas) y actúa como conector sintáctico.

(3) Transcripción: elf3_06 (línea 162). Participante: IAG. Edad: 3;3.06

*MON: está tocando la señal de prohibido pasar .

*IAG: sí .

*RIC: $\quad$ peo $\left[{ }^{*}\right][/]$ peo no puede pasar porque está rojo .

Todos los usos de porque detectados en el corpus han sido codificados partiendo de este sistema categorial tripartito, lo que permite obtener datos cuantitativos relativos a 
la frecuencia de cada construcción en el periodo examinado, resultados que exponemos en el siguiente apartado.

\section{Resultados}

En las más de 10.000 intervenciones analizadas hemos encontrado un total de 208 ocurrencias de porque. Comienza a ser manejado por los niños en torno a los 2;7 años, cuando su LME apenas supera las dos palabras por enunciado. Cabe destacar que, en todos los casos, este primer uso se corresponde con su utilización como conector pragmático, ya que como conector sintáctico no aparece, de media, hasta los 3;2 ańos, como se refleja en la Tabla 3.

Tabla 3. Datos Descriptivos de PORQUe

\begin{tabular}{|l|c|c|c|c|c|}
\hline Participante & Intervenciones & Usos & $\begin{array}{c}\text { LME de } \\
\text { primer uso }\end{array}$ & $\begin{array}{c}\text { Primer uso } \\
\text { pragmático }\end{array}$ & $\begin{array}{c}\text { Primer uso } \\
\text { sintáctico }\end{array}$ \\
\hline ANP & 1178 & $29(2,46 \%)$ & 2.475 & $2 ; 7.05$ & $3 ; 6.13$ \\
\hline CEC & 674 & $8(1,19 \%)$ & 1.891 & $2 ; 0.05$ & - \\
\hline ELI & 458 & $11(2,40 \%)$ & 1.895 & $2 ; 6.14$ & $3 ; 3.11$ \\
\hline IAG & 1483 & $31(2,09 \%)$ & 2.432 & $2 ; 10.05$ & $3 ; 4.13$ \\
\hline JOR & 1518 & $26(1,71 \%)$ & 3.076 & $2 ; 4.20$ & $2 ; 5.23$ \\
\hline NER & 562 & $4(0,71 \%)$ & 1.988 & $3 ; 0.16$ & $3 ; 6.23$ \\
\hline PAU & 380 & $4(1,05 \%)$ & 1.857 & $3 ; 4.13$ & - \\
\hline RIC & 1555 & $27(1,74 \%)$ & 1.580 & $2 ; 3.21$ & $3 ; 3.06$ \\
\hline XAC & 929 & $16(1,72 \%)$ & 1.946 & $2 ; 8.29$ & $3 ; 6.19$ \\
\hline XUN & 1438 & $52(3,62 \%)$ & 1.835 & $2 ; 1.15$ & $2 ; 4.27$ \\
\hline Total / media & 10175 & $208(2,04 \%)$ & 2.097 & $2 ; 7.05$ & $3 ; 2.06$ \\
\hline
\end{tabular}

Los valores pragmático-discursivos, además de ser los primeros en ser desempeñados por la partícula, son predominantes a lo largo del periodo cronológico examinado (2;0-4;0 años). A este respecto, en el Gráfico 1 se observa claramente el desequilibrio funcional que manifiestan los 208 usos analizados.

8. La aplicación del test Shapiro-Wilk a los resultados expuestos en esta columna $(\mathrm{p}=0.05)$ indica que la distribución de los datos es normal (W=0.91; p-value=0.28). 


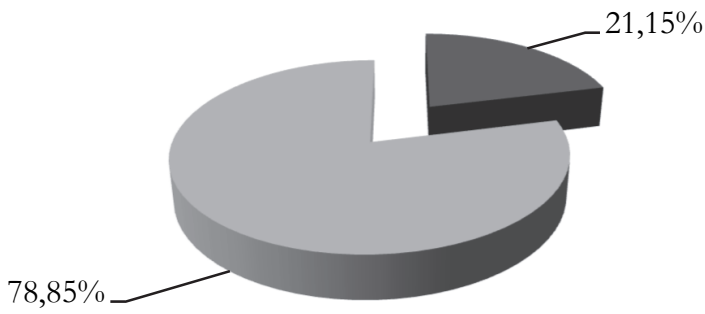

Usos sintácticos @ Usos pragmáticos

Gráfico i. Distribución de las fUnCIONES DE PORQUE.

Así pues, nuestros datos corroboran dos ideas bien conocidas por la bibliografía: el uso de porque desde temprana edad y su carácter predominantemente pragmático en el habla infantil (Aguado, 1995; Aparici et al., 2001; Prego Vázquez, 2010). Veamos qué implicaciones tiene esta situación en su utilización como enlace interclausular, que es lo que nos interesa. Para ello es conveniente caracterizar su nicho discursivo describiendo algunas propiedades relevantes.

En primer lugar, habida cuenta de su utilización como marcador, resulta interesante comprobar qué sucede en términos cohesivos. Los datos indican que porque está inicialmente destinado a cohesionar los enunciados infantiles, que encabeza, con una intervención adulta previa, contradiciendo la postura defendida por Bloom et al. (1980). No obstante, se perciben cambios a lo largo del periodo examinado, puesto que este tipo de usos descienden progresivamente, con un repunte en la fase final, como refleja el Gráfico 2.

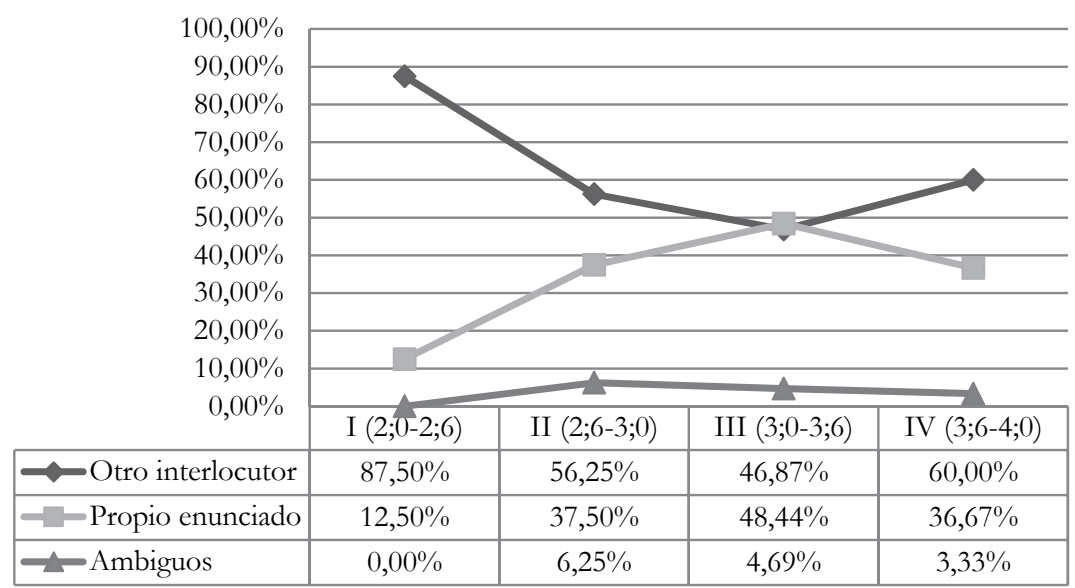

GrÁfICO 2. Tipo DE COHESIÓN POR ETAPAS. 
Lo habitual, por tanto, es que los niños empleen porque en contextos como los que representan los ejemplos (4) y (5), en los que el conector encabeza el turno infantil y lo vincula con un turno adulto inmediatamente precedente.

(4) Transcripción: elf4_04 (línea 263). Participante: RIC. Edad: 3;10.16

*MON: no tiene rayos el sol (.) no .

*RIC: $\quad$ porque no se las pusieron (.) sus rayas son rojas .

(5) Transcripción: bre6_10 (línea 434). Participante: CEC. Edad: 2;11.26

*PIL: $\quad$ se llama Ana y quién más tiene gafas a ver ¿

*CEC: yo también .

*PIL: $\quad$ y tú por qué no las traes ¿

*CEC: porque las tengo en casa .

El ejemplo (5), de hecho, ilustra un contexto en el que porque aparece repetidamente en el habla infantil: como marcador encabezando una respuesta ante una interrogativa adulta. Precisamente los datos reflejados en el Gráfico 3 muestran un progresivo descenso en el uso de porque como iniciador de una respuesta infantil ante una pregunta adulta, es decir, en el interior de un par de adyacencia.

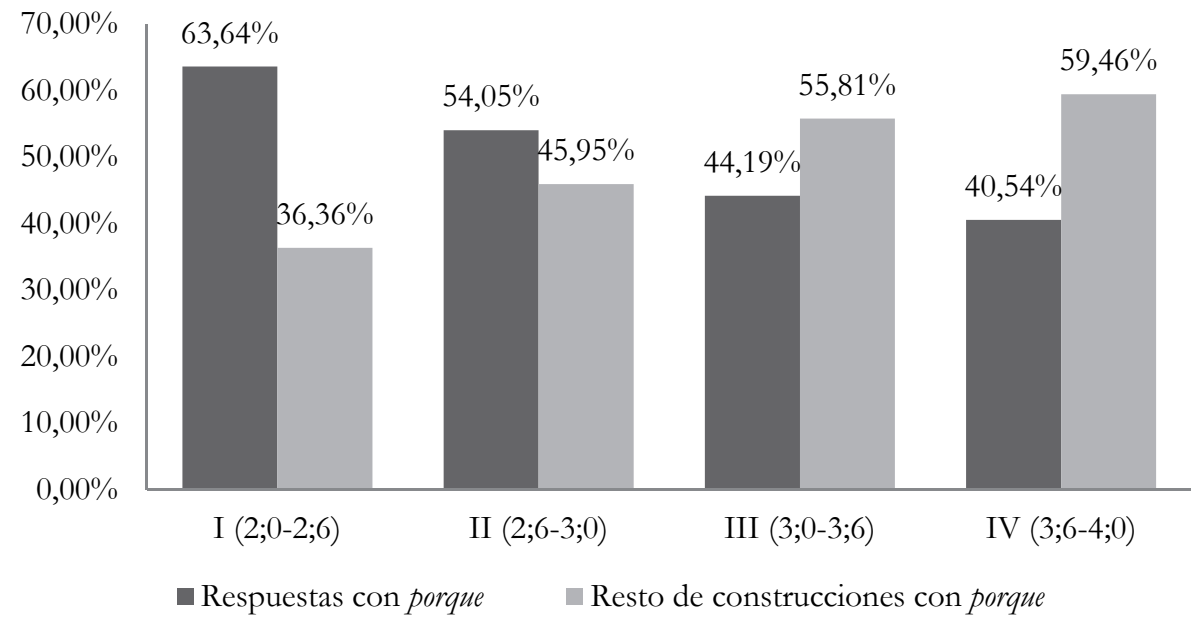

Gráfico 3. Usos de PORQUE EN PARES DE ADYACENCIA PREgUNTA-RESPUESTA.

Tanto el descenso del uso de porque para cohesionar con el discurso ajeno como en respuestas ante una interrogativa adulta son parámetros indicativos de una determinada tendencia evolutiva en lo que atańe a sus funciones. En concreto, los niños van alcanzando una mayor autonomía a la hora de emplear la partícula, es decir, progresivamente 
van ganando independencia con respecto al interlocutor adulto, circunstancia que se hace especialmente evidente a partir de los 3;0 años; al tiempo, van ampliando sus contextos de uso, pues al principio los conectores causales parecen restringidos a unas circunstancias concretas: encabezando respuestas ante una pregunta adulta (Braunwald, 1997; Diessel, 2004).

Estas características de uso del conector tienen implicaciones, como es lógico, en el plano formal, lo que nos conduce a retomar las categorías presentadas en la Tabla 2. Así, cuando porque actúa como marcador no aparece en estructuras que responden al modelo prototípico de las construcciones causales, sino que se sitúa en posición inicial absoluta del enunciado e introduce una única proposición, tal y como se puede comprobar en el ejemplo (6).

(6) Transcripción: elf4_07 (línea 71). Participante: IAG. Edad: 4;1.08

*MON: y no lo cogió .

*IAG: porque voló .

Como vemos, no tenemos dos cláusulas vinculadas por el nexo bajo un único contorno entonativo, sino que porque vincula unidades comunicativas diferentes, generalmente de interlocutores distintos —esto es, dos turnos- Esto da lugar a una construcción causal co-construida en la estructura vertical de la conversación que se parafrasearía, en este caso concreto, por y no lo cogió porque voló. Estas construcciones compartidas son las más frecuentes en las muestras analizadas, superando el $42 \%$, como se puede comprobar en el Gráfico 4.

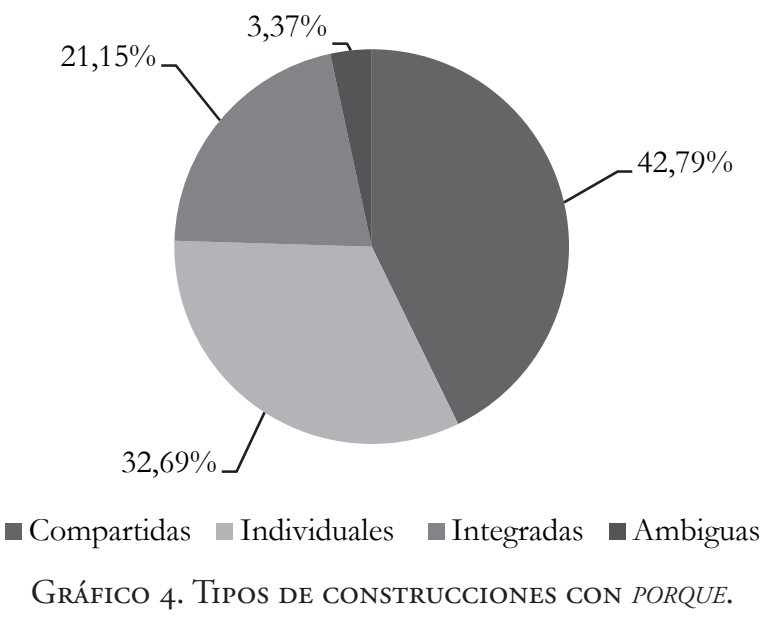

Los datos indican, pues, que porque suele aparecer en el habla infantil en intercambios en los que los nińos toman como apoyo la intervención del adulto para expresar una 
relación de causalidad entre dos unidades pragmáticas. Es decir, el nexo actúa como marcador discursivo que cohesiona proposiciones en la estructura vertical de la conversación, una tendencia ya identificada en el desarrollo gramatical temprano por autores como Ochs, Schieffelin y Platt (1979).

Ahora bien, cuando examinamos los datos desde el punto de vista evolutivo comprobamos que, a pesar de la predominancia de las construcciones compartidas, estas disminuyen progresivamente su frecuencia. De nuevo, además, parece que la frontera de los 3;0 años vuelve a marcar un punto de inflexión, como se ve en el Gráfico 5.

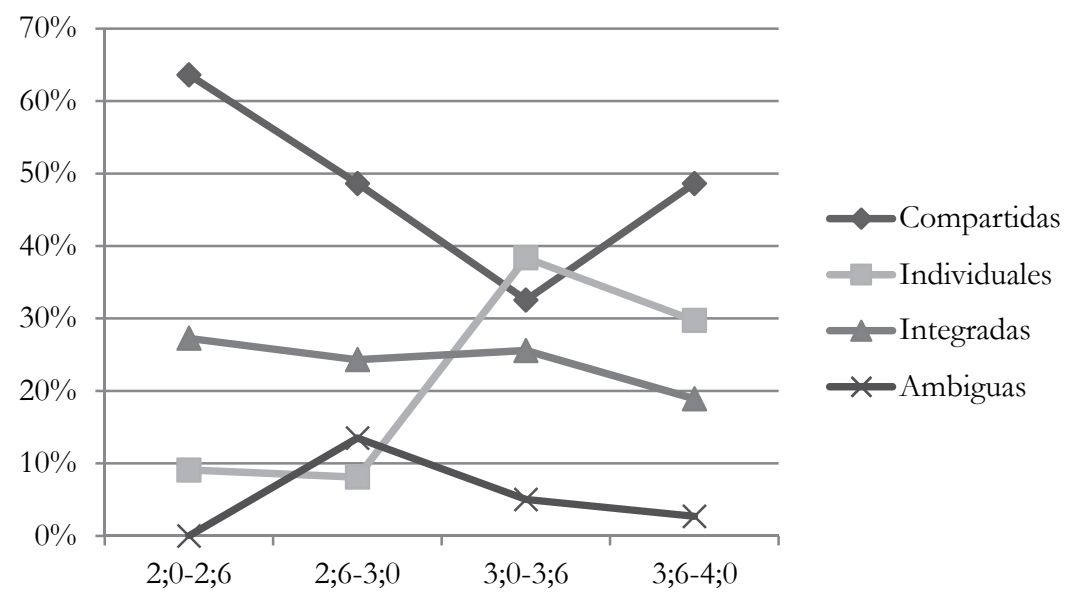

Gráfico 5. Tipos De CONSTRUCCIONES CON PORQUE POR ETAPAS.

El descenso de porque en estructuras coconstruidas con el adulto tiene como contrapartida un aumento en las construcciones individuales, hasta el punto de que incluso son las más utilizadas en un estadio $(3 ; 0-3 ; 6)$. En estas estructuras, que tenemos representadas en el ejemplo (7), porque sigue actuando como conector pragmático, si bien estos usos implican una mayor conciencia del nińo sobre la materia discursiva propia, de manera que el enlace vincula ya dos unidades comunicativas emitidas por él mismo?.

(7) Transcripción: elf4_05 (línea 198). Participante: RIC. Edad: 3;10.30

*RIC: $\quad$ Bea va a la playa.

*MON: muy bien (.) Bea va a la playa.

*RIC: $\quad$ porque aquí dentro tiene un flotador.

9. Esta tendencia, por tanto, es coherente con el aumento en el uso de porque para cohesionar enunciados propios (v. Gráfico 2). 
Cabe destacar también que, como sucede en (7), en estos contextos suele intercalarse entre las dos unidades pragmáticas conectadas una intervención del adulto. Este tipo de enunciados constituyen ejemplos muy claros de lo que se conoce como retroalimentación verbal, esto es, enunciados que tienen como función implícita reforzar, modelar y corregir el habla del niño de acuerdo con las convenciones de la lengua adulta (Rondal, 1983). No obstante, consideramos que, junto a esta función, cuando se ven implicadas construcciones con porque, las intervenciones adultas ayudan también a que el niño prosiga con su discurso y, por consiguiente, acabe vinculando sus enunciados mediante la partícula. Esta circunstancia, ya descrita en la bibliografía especializada (Rojas Nieto, 1992), se cristaliza en el denominado lenguaje contingente, caracterizado por la reiteración en el uso de las interrogativas, como sucede en el ejemplo (8).

(8) Transcripción: vit4_05 (línea 330). Participante: XUN. Edad: 3;4.03

*XUN: estoy enfada(d)o contigo .

*ANC: $\quad$ por qué $\dot{ }$

*XUN: porque no me toca animal.

El discurso del niño podría haber finalizado con su primera intervención; sin embargo, la pregunta del adulto propicia que continúe hablando y, en consecuencia, que establezca una relación de tipo causal entre las proposiciones expresadas a través de la estructura discursiva: estoy enfadado contigo porque no me toca animal. El niño entra en contacto así, a través del diálogo, con el discurso multi-proposicional, dado que a estas edades todavía es extraña la codificación de relación interclausulares.

En este sentido, las estructuras que representan el modelo prototípico de construcción compleja causal, las construcciones integradas, tienen una escasa presencia en los datos. Estas construcciones apenas superan el $21 \%$ del total y se mantienen siempre en una frecuencia reducida, ya que nunca llegan a alcanzar, en ninguno de los cuatro estadios, el 30\%. En estas circunstancias, y a diferencia de lo que ocurre en las dos categorías anteriores, porque actúa como nexo gramatical que conecta dos unidades gramaticales en el mismo contorno entonativo, expresando una relación sintáctica entre dos cláusulas. Los ejemplos (9) y (10) responden a este modelo.

(9) Transcripción: vit4_01 (línea 90). Participante: JOR. Edad: 3;2.26

*JOR: pues yo fui a ver Tarzán y me levanté um@i porque me iba a sentar bien .

(10) Transcripción: vit4_01 (línea 470). Participante: XUN. Edad: 3;1.16

*XUN: pues yo cuando era pequeño me puse aquí una tirita $<$ porque $>[/]$ porque me $<$ han quemado $\left.>{ }^{*}\right]$ con un pitillo y con fuego .

A pesar de su escasez, consideramos interesante observar qué tipo de causalidad expresa porque entre ambas proposiciones: física, en la que se manifiesta directamente 
una relación causal de la realidad, o psicológica, en la que se expresa indirectamente, al codificar relaciones causales de tipo cognitivo, en el mundo mental del individuo. A este respecto, algunas investigaciones centradas en el inglés desmintieron una idea hasta entonces bastante extendida en el campo, puesto que, en contra de lo que se creía, demostraron que la causalidad psicológica se desarrolla antes que la física (Hood y Bloom, 1979; McCabe y Peterson, 1985). Resulta fundamental conocer si lo mismo sucede en español, dado que los materiales clínicos y pedagógicos recurren habitualmente a las construcciones causales y a las interrogativas como ítems con los que valorar y evaluar el desarrollo lingüístico de los niños.

Así pues, los 44 usos de porque como conector sintáctico se distribuyen, en lo que tiene que ver con el tipo de causalidad, tal y como refleja la Tabla 4.

Tabla 4. Tipo de causalidad

\begin{tabular}{|l|c|c|c|}
\hline Participante & $\begin{array}{c}\text { Causalidad } \\
\text { física }\end{array}$ & $\begin{array}{c}\text { Causalidad } \\
\text { psicológica }\end{array}$ & $\begin{array}{c}\text { Usos sintácticos } \\
\text { de porque }\end{array}$ \\
\hline ANP & 0 & 1 & 1 \\
\hline CEC & 0 & 0 & 0 \\
\hline ELI & 0 & 1 & 1 \\
\hline IAG & 0 & 1 & 1 \\
\hline JOR & 2 & 7 & 9 \\
\hline NER & 1 & 0 & 1 \\
\hline PAU & 0 & 0 & 0 \\
\hline RIC & 2 & 4 & 6 \\
\hline XAC & 4 & 2 & 6 \\
\hline XUN & 6 & 13 & 19 \\
\hline $\begin{array}{l}\text { Total } / \\
\text { frecuencia }\end{array}$ & 15 & 29 & 44 \\
\hline
\end{tabular}

Como se puede observar, nuestros datos indican que también en español lo habitual en el habla infantil es el uso de porque para codificar causalidad psicológica, como en el ejemplo (11).

(11) Transcripción: vit3b_07 (línea 498). Participante: XUN. Edad: 2;11.15

*XUN: hoy no hay porque soy bueno y hoy duermo mucho. 
En cambio, su utilización para expresar causalidad física, uso representado por el ejemplo (12), es menos frecuente, a pesar de que, en principio, podría parecer cognitivamente más simple.

(12) Transcripción: vit4_04 (línea 506). Participante: XUN. Edad: 3;3.06 *XUN: me metió en un vaso la avispa porque quería comer la cocacola .

Esta circunstancia resulta indicativa de la semántica expresada por porque en su utilización temprana como nexo gramatical, y ha de ser tenida en cuenta a la hora de elaborar materiales destinados a evaluar y mejorar las capacidades comunicativas de los niños.

\section{Conclusiones}

El análisis de los datos proporcionados por el corpus Koiné permite corroborar que porque es un conector temprano, que aparece en el habla infantil en torno a los 2;7 años. Estos resultados coinciden con los alcanzados en estudios previos sobre el desarrollo del español (Aguado, 1995; Hernández-Pina, 1984), así como sobre conectores equivalentes en otras lenguas, donde también se ha constatado su utilización antes de los 3;0 ańos (Bloom et al., 1980; Braunwald, 1997; Diessel, 2004; Evers-Vermeul y Sanders, 2011; Sekali, 2012). Ahora bien, la aparición de la forma marca solamente el punto de partida, ya que el dominio de porque constituye un proceso en el que se van integrando gradualmente sus funciones (Prego Vázquez, 2010). Así, hemos constatado que al principio los niños lo utilizan sobre todo como marcador (Díez-Itza y Pérez Toral, 2001), puesto que les permite gestionar sus intervenciones en el diálogo, cohesionando sus enunciados con los de su interlocutor —en contra de la postura defendida por Bloom et al. (1980)—, e introduciendo respuestas ante una interrogativa adulta. En cambio, su utilización como conector sintáctico, poco frecuente en el periodo examinado, no se detecta hasta aproximadamente los 3;2 años.

Así pues, podemos afirmar que porque, como pero (Varela, 2011), es incorporado por los niños en la interacción dialógica, a través de un proceso recíproco en el discurso (Braunwald, 1997), de ahí que sea utilizado predominantemente en estructuras coconstruidas con el adulto. No obstante, esta estrategia de producción compartida desciende conforme avanza el desarrollo, lo cual puede relacionarse con la incidencia variable que el input parental tiene en estas fases tempranas (van Veen et al., 2009, 2013). En un sentido parecido, el descenso de la frecuencia de porque en pares de adyacencia pregunta-respuesta, también detectado en inglés (Diessel, 2004, pp. 160163), apunta a un nicho interaccional y discursivo concreto, en el cual radica el origen ontogenético de la partícula. Al principio, pues, los niños restringen el uso de porque a unas circunstancias muy específicas y poco a poco irán ampliando sus contextos de uso (Braunwald, 1997). Todo ello indica que el interlocutor adulto y la práctica de 
las habilidades conversacionales son fundamentales para el adecuado desarrollo de la complejidad sintáctica, pues ambos factores permiten al niño ir ganando autonomía en la producción de estructuras causales. Así, a partir de los 3;0 años, y con este bagaje detrás, se inicia lo que se conoce como proceso de vinculación de las cláusulas (Diessel, 2004; Varela, 2011). Hemos podido constatar, además, que estas primeras construcciones complejas con porque codifican relaciones de causalidad de tipo psicológico con mayor frecuencia que las de tipo físico, contradiciendo así una idea relativamente extendida en el campo (Hood y Bloom, 1979; McCabe y Peterson, 1985).

Los resultados obtenidos, además de mostrar que el desarrollo de porque es similar al de enlaces equivalentes en otras lenguas, traen a colación un par de ideas particularmente importantes por sus implicaciones teóricas y prácticas. En primer lugar, es necesario subrayar que el uso reiterado de porque en el habla infantil no significa necesariamente que su proceso de adquisición haya finalizado, ya que las diferentes funciones del conector se van incorporando progresivamente al repertorio comunicativo del nińo. El desarrollo de las construcciones causales es un proceso gradual y complejo, en el que se ven interrelacionados factores cognitivos, sociales y lingüísticos (Braunwald, 1997) y que comienza poco después de los 2;0 años, con los primeros usos pragmáticos de porque. Los datos indican que existen ciertos precedentes discursivos que constituyen la base sobre la que se asentará la función sintáctica de la partícula, apenas unos meses más tarde (Prego Vázquez, 2010).

Por otro lado, aunque en evidente relación, se ha comprobado que las estructuras causales se desarrollan a partir del contexto conversacional y mediante un proceso de integración, como sucede en inglés (Diessel, 2004, pp. 169-173) o en francés (Sekali, 2012), dado que los niños se apoyan inicialmente en los enunciados adultos para expresar relaciones de causalidad (Aparici et al., 2001), dando lugar a construcciones compartidas (Givón, 2009). Hay que poner en valor, por ello, la importancia evolutiva de las construcciones erróneamente calificadas por algunos autores como incompletas (Kyratzis, Guo y Ervin-Tripp, 1990) o fragmentarias (Tyack y Gottsleben, 1986), al considerar que falta la cláusula principal de la estructura compleja ${ }^{10}$. Este tipo de construcciones constituyen, como hemos querido demostrar con este trabajo, el punto de partida del proceso de desarrollo de las construcciones causales.

En definitiva, a la hora de describir el habla del niño y evaluar su desarrollo hay que tener presente que es posible que use frecuentemente porque y, sin embargo, que todavía no sea capaz de producir una construcción compleja causal. Los niños comienzan utilizando este conector no como nexo gramatical en estructuras causales, sino como marcador discursivo. Estas circunstancias han de tenerse en cuenta a la hora de elaborar materiales clínicos y pedagógicos efectivos, así como en el diseño de actividades en las aulas de educación infantil. Solamente una aproximación pragmático-discursiva al habla

10. Es decir, solamente son "incompletas" cuando las analizamos desde un punto de vista estrictamente sintáctico; el enfoque pragmático, en cambio, revela que están perfectamente construidas. 
infantil permite, pues, captar todos los factores implicados en el complejo proceso de desarrollo de la lengua materna (Fernández Pérez, 2014).

\section{Referencias}

Aguado, G. (1995). El desarrollo del lenguaje de 0 a 3 años. Madrid: Cepe.

Aguado, G. (1988). Valoración de la competencia morfosintáctica en el niño de dos años y medio. Infancia y Aprendizaje, 43, 73-96.

Alfaro, P., Crespo, N., y Alvarado, C. (2016). Complejidad sintáctica en narraciones de niños con desarrollo típico, trastorno específico del lenguaje y discapacidad intelectual. Sintagma, 28, 27-41.

Aparici, M., Capdevila, M., Serrat, E., y Serra, M. (2001). Acquisition of complex sentences in Spanish and Catalan speaking children. En K. E. Nelson, C. E. Johnson y A. Aksu-Koç (Eds.), Children's Language (pp. 1-25). Hillsdale: Lawrence Erlbaum Associates.

Bloom, L., Lahey, M., Hood, L., Lifter, K., y Fliess, K. (1980). Complex sentences: Acquisition of syntactic connectives and the semantics relations they encode. Journal of Child Language, 7, 235-261.

Braunwald, S. R. (1997). The development of because and so: Connecting language, thought, and social understanding. En J. Costermans y M. Fayol (Eds.), Processing interclausal relationships: Studies in the production and comprehension of text (pp. 121137). Nueva Jersey: Erlbaum.

Brown, R. (1973). A first language: The early stages. Cambridge: Harvard University Press.

Briz, A. (2000). Las unidades de la conversación. RILCE, 16(2), 225-246.

CHILDES = MacWhinney y Snow (1985).

Clemente, R. A. (1989). Medida del desarrollo morfosintáctico. Los problemas de la medición y la utilización de la MLE. Anuario de Psicología, 42, 103-113.

Diessel, H. (2004). The acquisition of complex sentences. Cambridge: Cambridge University Press.

Díez-Itza, E., y Pérez Toral, M. (2001). Categorización funcional de los marcadores discursivos en narraciones infantiles orales. En A. I. Moreno y V. Colwell (Eds.), Perspectivas recientes sobre el discurso. León: AESLA/Universidad de León [Publicación en CD].

Díez-Itza, E., Snow, C., y MacWhinney, B. (1999). La metodología RETAMHE y el proyecto CHILDES: Breviario para la codificación y análisis del lenguaje infantil. Psicothema, 11(3), 517-530.

Evers-Vermeul, J., y Sanders, T. (2011). Discovering domains. On the acquisition of causal connectives. Journal of Pragmatics, 43(6), 1645-1662.

Fernández Pérez, M. (2006). Corpus Koiné de habla infantil. Universidade de Santiago de Compostela. Recuperado de: https://phonbank.talkbank.org/access/Spanish/koine.html 
Fernández Pérez, M. (2011). Lingüistica de corpus y adquisición de la lengua. Madrid: Arco Libros.

Fernández Pérez, M. (2014). Lingüistica y déficit comunicativos. ¿Cómo abordar las disfunciones verbales? Madrid: Síntesis.

Givón, T. (2009). The énesis of syntactic complexity: Diachrony, ontogeny, neurocognition, evolution. Ámsterdam: John Benjamins.

Hernández-Pina, F. (1984). Teorías psicolingüisticas y su aplicación a la adquisición del español como lengua materna. Madrid: Siglo XXI.

Hoff, E. (2014). Language development ( 5 a ed.). Wadsworth: Cengage.

Hood, L., y Bloom, L. (1979). What, when, and how about why: A longitudinal study of expressions of causality in the language development of two-year-old children. Monographs of the Society for Research in Child Development, 44(6), 1-47.

Koiné = Fernández Pérez, M. (2006)

Kyratzis, A., Guo, J., y Ervin-Tripp, S. (1990). Pragmatic conventions influencing children's use of causal constructions in natural discourse. En D. J. Costa (Ed.), Proceedings of the Sixteenth Annual Meeting of the Berkeley Linguistics Society (pp. 205214). Berkeley: University of California.

MacWhinney, B. (2000). The CHILDES Project: Tools for analyzing talks (3a ed.) Mahwah, NJ: Lawrence Erlbaum Associates.

MacWhinney, B., y Snow, C. (1985). The Child Language Data Exchange System. Journal of Child Language, 12(2), 271-296.

McCabe, A. E., y Peterson, C. (1985). A naturalistic study of the production of causal connectives by children. Journal of Child Language, 12(1), 145-159.

Ochs, E., Schieffelin, B. B., y Platt, M. L. (1979). Propositions across utterances and speakers. En E. Ochs y B. B. Schieffelin (Eds.), Developmental pragmatics (pp. 251268). Nueva York: Academic Press.

Prego Vázquez, G. (2006). Evaluación de la cohesión en el habla infantil: Los usos de $y$ en edad temprana. En C. Hernández Sacristán, B. Gallardo y V. Moreno (Eds.), Actas del I Congreso Nacional de Lingüistica Clínica (pp. 265-279). Valencia: Universitat de València.

Prego Vázquez, G. (2010). Los marcadores explicativos en edad temprana: Usos y etapas evolutivas de porque en el corpus Koiné. En B. Gallardo y V. Moreno (Eds.), Estudios de lingüistica clínica (pp. 179-202). Valencia: Universitat de València.

Rojas Nieto, C. (1992). La pregunta en la construcción del diálogo. Funciones constructivas de las preguntas a los niños pequeños. Revista de Lingüistica Aplicada, 15/16, 182-198.

Rondal, J. A. (1983). L'interaction adulte-enfant et la construction du langage. Bruselas: Pierre Mardiaga.

Sebastián, E., y Slobin, D. I. (1994). Development of linguistic forms: Spanish. En R. E. Berman y D. I. Slobin (Eds.), Relating events in narrative: A crosslinguistic developmental study (pp. 239-284). Hillsdale: Lawrence Erlbaum Associates. 
Sekali, M. (2012). The emergence of complex sentences in a French child's language from $0 ; 10$ to 4;01: A causal adverbial clauses and the concertina effect. Journal of French Language Studies, 22(1), 115-141.

Tomasello, M. (2003). Constructing a language. A usage-based theory of language acquisition. Cambridge: Harvard University Press.

Tyack, D. L., y Gottsleben, R. H. (1986). Acquisition of complex sentences. Language, Speech, and Hearing Services in Schools, 17, 160-174.

van Veen, R., Evers-Vermeul, J., Sanders, T., y van den Bergh, H. (2009). Parental input and connective acquisition: A growth curve analysis. First Language, 29(3), 266-288.

van Veen, R., Evers-Vermeul, J., Sanders, T., y van den Bergh, H. (2013). The influence of input on connective acquisition: A growth curve analysis of English because and German weil. Journal of Child Language, 40(5), 1003-1031.

Varela, V. (2011). El nicho discursivo de las construcciones infantiles con pero en la adquisición temprana del español. En C. Rojas y D. Jackson-Maldonado (Eds.), Interacción y uso lingüistico en el desarrollo de la lengua (pp. 173-208). México: Instituto de Investigaciones Filológicas, Universidad Nacional Autónoma de México y Universidad de Querétaro. 